\title{
Prediction of Left Main Coronary Artery Occlusion by ST Segment Elevation in Lead aVR Greater than that in Lead $V_{1}$ in Acute Coronary Syndrome
}

\author{
Abdul Azeez Ahemd ${ }^{1}$, Mahboob $\mathrm{Ali}^{2}$, Abdullah Al Shafi Majumder ${ }^{2}$, M. Atahar $\mathrm{Ali}^{2}$, Md. Shafiqur \\ Rahman Patwary ${ }^{2}$, Mohammad Arifur Rahman² \\ ${ }^{1}$ Indhira Gandhi Memorial Hospital, Male, ${ }^{2}$ National Institute of Cardiovascular Diseases, Dhaka
}

Keywords:

Left main

coronary artery

disease, $S T$

segment, lead

aVR, Acute

coronary

syndrome.

\begin{abstract}
Background: The electrocardiogram (ECG) predicting an acute obstruction of the LMCA, which requires immediate aggressive treatment, is very important for early diagnosis. We correlated ST segment elevation in lead aVR greater than that in lead $V \cdot$ with coronary angiographic diagnosis of LMCA occlusion in patients with acute coronary syndrome.

Methods: Cross sectional analytical study was conducted in the Department of Cardiology, National Institute of Cardiovascular Diseases (NICVD), Dhaka, Bangladesh from August 2011 to July 2012. Total 90 patients were included purposively. Study population was divided into two groups. Group I- Patients with ST segment elevation in aVR greater than ST segment elevation in $V \cdot(n=45)$ and group II- Patients with ST segment elevation in aVR less than that in lead V • (n=45). In hospital outcomes were observed for cardiogenic shock, left ventricular failure, hypotension, arrhythmia and death.

Results: Acute LVF was significantly $(P<0.05)$ higher in group I but other complications were not significant $(P>0.05)$ between two groups. LM involvement was significantly higher in group I (91.1\% vs. $20.0 \%, p<0.05)$. ST segment elevation in aVR greater than ST segment elevation in $V \cdot(n=45)$ for prediction of LM significant disease has got a sensitivity of $82.0 \%$, specificity $90.0 \%$, accuracy $85.6 \%$, positive and negative predictive values were $91.1 \%$ and $80.0 \%$ respectively.

Conclusion: ST segment deviation in lead aVR greater than that in lead V1 is supposed to be a positive predictor of left main coronary artery obstruction with highly sensitivity and accuracy. Precordial leads V1 and V6 can also predict the critical LMCA obstruction in patients with acute coronary syndrome.
\end{abstract}

(Cardiovasc. j. 2017; 9(2): 77-82)

\section{Introduction:}

Left main coronary artery occlusion is a serious clinical condition. Despite its low incidence, the prognosis is grave. It may present as sudden death, complete heart block, shock and/or acute coronary syndrome. Surgery is usually too late to initiate, so that percutaneous coronary intervention (PCI) is utilized to obtain immediate vessel patency. However, this modality is associated with a high mortality and restenosis rate. Early recognition and emergent PCI may be lifesaving. ${ }^{1}$ By far the most common etiology of LMCA disease is atherosclerosis. ${ }^{2}$

An acute obstruction of left main coronary artery (LMCA) is encountered approximately in only $0.5 \%$ of acute myocardial infarction (AMI) cases. The ECG predicting an acute obstruction of the LMCA, which requires immediate aggressive treatment, is very important for early diagnosis. Various studies have showed that ECG findings of acute LMCA occlusion may be different and confusing. ${ }^{3}$

Address of Correspondence: Dr. Mohammad Arifur Rahman, Registrar, Cardiology, National Institute of Cardiovascular Diseases, Dhaka, Bangladesh. Mobile- email-drarif79@yahoo.com 
LM disease is usually not an isolated coronary artery event, but is associated with individual LAD, LCX, RCA or even triple-vessel disease. Cooccurrence with one or more coronary arteries may alter ECG manifestations, as the magnitude of ST elevation in lead aVR may change in relation to $V_{1}$. Diffuse $S T$ depression over the inferior and anteriolateral leads with ST elevation in leads aVR, aVL, $V_{1}$ and $V_{2}$ are typical ECG manifestations of AMI secondary to an LM occlusion. ECG is only one of the many diagnostic modalities used in detecting myocardial infarction from an LM occlusion. Therefore, recognizing the significance of ST elevation in lead aVR and subjecting the patient to emergent cardiac catheterization are lifesaving. ${ }^{1}$

ST elevation in lead aVR strongly suggests a significant LM lesion. However, if accompanied by ST elevation in lead $V_{1}$, the specificity of an LM lesion acting as the culprit vessel increases. Assuming that both leads aVR and $\mathrm{V}_{1}$ have ST elevations, a higher magnitude of ST elevation in lead aVR compared to lead $V_{1}$ is very specific for LM occlusion. For the past few years, reports have shown that the ST segment deviation in lead aVR is an important predictor of acute LM occlusion, and is valuable in its prognosis. Also, an ST elevation of more than $0.05 \mathrm{mV}$ in lead aVR is indicative of LM occlusion in up to $88 \%$ of patients with acute myocardial infarction. ${ }^{1}$

Lead aVR can be very helpful in ischemic heart disease in diagnosing the site of coronary artery occlusion and size of the area at risk. In patients with unstable angina we found that ST-segment changes in eight leads, including ST-elevation in lead aVR, are highly predictive for left main or three-vessel disease. ${ }^{4}$

lead aVR ST elevation accompanied by either anterior ST elevation or widespread ST-segment depression may indicate left main occlusion. ${ }^{5} \mathrm{It}$ is strongly believed that ST elevation in lead aVR with accompanying ST elevation in lead $\mathrm{V} \cdot$ of lower magnitude than aVR increases the specificity (100\%) and positive predictive value (100\%) for pointing out the LMCA as the culprit vessel in ACS. The incidence of ST elevation in lead aVR of lower magnitude or less than that of $\mathrm{V} \cdot$ in AMI from LM occlusion is less than $20 \%{ }^{6}$

Thus the simple non-invasive, inexpensive, easily available ECG can help us earlier prediction of the deadly lesion in LMCA, so that we can proceed further to confirm the diagnosis and can take prompt, appropriate treatment strategy to reduce the mortality in acute coronary syndrome.

\section{Methods:}

Cross sectional analytical study was conducted in the Department of Cardiology, NICVD, Dhaka, Bangladesh from August 2011 to July 2012. Objectives of the study were to correlate ST segment elevation in lead aVR greater than that in lead $V_{1}$ with coronary angiographic findings and to assess the predictive value of ST segment elevation in lead aVR greater than the ST segment elevation in lead $V_{1}$ in the diagnosis of LMCA occlusion in patients with acute coronary syndrome. Significant left main coronary artery stenosis were considered when it was $>50 \%$ stenosis. Total 90 patients were included purposively. Study population was divided into two groups. Group I- Patients with ST segment elevation in aVR greater than ST segment elevation in $\mathrm{V}_{1} \quad(n=45)$ and group IIPatients with ST segment elevation in aVR less than that in lead $V_{1}(n=45)$. In hospital outcomes were observed for cardiogenic shock, left ventricular failure, hypotension, arrhythmia and death.

\section{Statistical Methods:}

The numerical data obtained from the study were analyzed and significance of differences was estimated by using statistical methods. Computer based SPSS was used for all the analysis. Data were expressed in percentage, frequencies and means and standard deviation as applicable. Comparison between two groups was done by Student's t-test, chi-square test as applicable. P value of less than 0.05 was considered as significant. Comparison between 15 lead ECG findings and coronary angiographic findings were done by statistical test of accuracy. (Sensitivity, specificity, predictive value using the $2 \times 2$ table method of validity test. 


\section{Results:}

Table-I

Demographic characteristics of study population $(n=90)$.

\begin{tabular}{lccc}
\hline & Group I & Group II & p value \\
\hline Age(M \pm SD) & $53.58 \pm 8.0$ & $52.6 \pm 8.9 .59$ & 0.60 \\
Male sex & $39(86.7)$ & $38(84.4)$ & 0.76 \\
Pulse (M \pm SD) & $76.86 \pm 6.96$ & $76.63 \pm 6.96$ & 0.88 \\
BP systolic & $121.36 \pm 14.4$ & $126.74 \pm 14.59$ & 0.08 \\
BP diastolic & $73.86 \pm 8.41$ & $74.51 \pm 7.16$ & 0.69 \\
Smoking & $31(68.9)$ & $39(86.7)$ & 0.04 \\
Hypertension & $24(53.3)$ & $24(53.3)$ & 0.53 \\
Diabetes & $21(46.7)$ & $21(46.7)$ & \\
Dyslipidaemia & $9(20)$ & $3(6.7)$ & \\
F/H of IHD & $11.1(88.9)$ & $7(15.6)$ & 0.16 \\
UA & $16(35.6)$ & $10(22.2)$ & 0.15 \\
NSTEMI & $10(22.2)$ & $5(11.1)$ & 0.02 \\
STEMI & $19(42.2)$ & $30(66.7)$ & 0.12 \\
Hypotension & $3(6.7)$ & 0 & 0.003 \\
Acute LVF & $10(22.2)$ & $1(2.2)$ & 0.12 \\
Cardiogenic shock & $3(6.7)$ & 0 & 0.12 \\
Arrhythmia & $3(6.7)$ & 0 & \\
Death & 0 & 0 & 0.83 \\
Anterior & $19(42.2)$ & $24(53.3)$ & 0.50 \\
Inferior & $4(8.9)$ & $3(6.7)$ &
\end{tabular}

Table-II

Distribution of the study patients according to coronary artery with site of occlusion $(n=90)$.

\begin{tabular}{lccc}
\hline Vessel involved & Group In (\%) & Group IIn (\%) & p value \\
\hline LM & $41(91.1)$ & $9(20)$ & .001 \\
LAD & $21(46.7)$ & $31(68.9)$ & .032 \\
LCX & $17(37.8)$ & $16(35.6)$ & .826 \\
RCA & $24(53.3)$ & $18(40)$ & .204 \\
\hline
\end{tabular}

Table-III

Comparison between ECG and angiogram for prediction of $L M(n=90)$.

\begin{tabular}{lccc}
\hline ECG & \multicolumn{2}{c}{ Angiographic evaluation } & Total \\
& Positive for LM & Negative for LM & \\
\hline Suspected case of LM positive & $41(\mathrm{TP})$ & $4(\mathrm{FP})$ & 45 \\
Suspected case of LM negative & $9(\mathrm{FN})$ & $36(\mathrm{TN})$ & 45 \\
\hline Total & 50 & 40 & 90 \\
\hline
\end{tabular}


Table-IV

Sensitivity, specificity, accuracy, positive and negative predictive values of the ECG evaluation for prediction of LM significant disease.

\begin{tabular}{lc}
\hline Validity test & Percentage \\
\hline Sensitivity & $82.0 \%$ \\
Specificity & $90.0 \%$ \\
Accuracy & $85.6 \%$ \\
Positive predictive value & $91.1 \%$ \\
Negative predictive value & $80.0 \%$ \\
\hline
\end{tabular}

\section{Discussion:}

The mean age was found $53.58 \pm 8.0$ years in group I and 52.6 \pm 9.59 years in group II, which was almost similar between two groups. Ockene, et al. observed the mean age of coronary artery disease patients was 49 years with range from $20-70$ years. ${ }^{7}$ Gonzalez-Porras, et al. observed that the mean age of patients was 47 years with range from $26-54$ years. ${ }^{8}$ The sex incidence of the present study it was observed that male was found $86.7 \%$ in group I and $84.4 \%$ in group II. Male to female ratio was almost $6: 1$ which was closely resembled with previous study where the authors found male female ratio almost 6:1. Similarly other studies mentioned that Coronary artery disease (CAD) affects male more frequently and severely than female. ${ }^{8-10}$

Regarding the risk factors, it was observed in this series that smoker was $68.9 \%$ in group I and $86.7 \%$ in group II. Hypertension observed 53.3\% and $53.3 \%$ in group I and group II respectively. Diabetes was found $46.7 \%$ in group I and $46.7 \%$ in group II. Dyslipidaemia were in $20.0 \%$ and $6.7 \%$ in group I and group II respectively. Smoker was significantly $(p<0.05)$ higher in group II but others risk factors were almost similar between two group. Puri et al. showed that hypertension, smoking and dyslipidaemia were the most common risk factors in patients with CAD. Similarly Yildirim et al. \& Avezum et al., reported that HTN, smoking and dyslipidemia were most frequent risk factors in patients with ACS. ${ }^{11,12}$

Regarding the clinical presentation STEMI was found $42.2 \%$ and $66.7 \%$ in group I and group II respectively. STEMI was significantly $(p<0.05)$ higher in group II but others (NSTEMI and UA) were not significant $(p>0.05)$ between two groups.
Heart failure was found $8(17.8 \%)$ in group I and $2(4.4 \%)$ in group II. I class heart failure was found $3(6.7 \%)$ and $2(4.4 \%)$ in group I and group II respectively. II and III class heart failure was found $3(6.7 \%)$ and $2(4.4 \%)$ in group I but not found in group II. The difference was not statistically significant $(p>0.05)$ between two groups. Regarding the risk factors, smoker was found $68.9 \%$ in group I and $86.7 \%$ in group II. Hypertension was found $53.3 \%$ and $53.3 \%$ in group I and group II respectively. Diabetes was $46.7 \%$ in group I and $46.7 \%$ in group II. Dyslipidaemia was found $20.0 \%$ and $6.7 \%$ in group I and group II respectively. Smoker was significantly $(\mathrm{p}<0.05)$ higher in group II but others risk factors were not significant ( $>0.05$ ) between two groups.

About the complications, hypotension $6.7 \%$, cardiogenic shock $6.7 \%$ and arrhythmias $6.7 \%$ in group I but not found in group II. Acute LVF was found $22.2 \%$ in group I and $2.2 \%$ in group II. Acute LVF was significantly $(p<0.05)$ higher in group I but others complications were not significant $(p>0.05)$ between two groups. The mean percentage of ejection fraction was found $54.94 \pm 7.69$ in group I and $53.93 \pm 6.37$ in group II. Mean percent of ejection fraction of was not statistically significant $(p>0.05)$ between two groups. On the contrary, the mean of ejection fraction $30-40 \%(8.9 \%)$ and $>60 \%$ (26.7\%) were higher in group I patients than group II patients $(2.2 \%)$ and $17.8 \%$ respectively.

LM involved was found $91.1 \%$ in group I and $20.0 \%$ in group II. LAD was $46.7 \%$ and $68.9 \%$ in group I and group II respectively. CCX was $37.8 \%$ in group I and $35.6 \%$ in group II. RCA was $53.3 \%$ in group I and $40.0 \%$ in group II. LM and LAD were significantly $(\mathrm{p}<0.05)$ higher in group I and group II respectively between two groups.

In patients with LMCA, ST segment elevation in lead aVR was two times more frequent than in remaining patients $(69.6 \%$ vs $34.6 \% \mathrm{p}=0.0001)$ whereas there were no differences in lead V1. ${ }^{13}$ ST-segment elevation in lead aVR of $\geq 0.5 \mathrm{~mm}$ was the strongest predictor of LM/3-VD. ${ }^{14}$ In-hospital mortality was $19 \%$ in the aVR(+) group and $5 \%$ in the aVR(-) group aVR positivity was an independent predictor of in-hospital death. ${ }^{15}$ The outcome measure was 30-day mortality. ST elevation in aVR $\geq 1 \mathrm{~mm}$ was associated with higher 30 -day mortality for both inferior (22.5\% for $\geq 1.5 \mathrm{~mm}$ and $13.2 \%$ for 
$1 \mathrm{~mm}$ ) and anterior (23.5\% for e"1.5 $\mathrm{mm}$ and $11.5 \%$ for $1 \mathrm{~mm}$ ) infarction. ${ }^{16}$

Uthamalingam et al. found that exercise treadmill testing (ETT) - induced ST-segment elevation (STE) in electrocardiographic lead aVR is an important indicator of significant left main coronary artery (LMCA). ${ }^{17}$ Current ACC/AHA guidelines state that "the benefit of surgery over medical treatment in patients with significant LMS stenosis (greater than $50 \%)$ is little argued. ${ }^{18}$

ECG evaluation of patients with ST segment elevation in aVR greater than ST segment elevation in $V_{1}(n=45)$ in comparison with ST segment elevation in aVR less than that in lead $V_{1}(n=45)$ out of the 90 cases, true positive 41 and false positive 4 , false negative 9 and true negative were 36 cases and the sensitivity was found $82.0 \%$, specificity $90.0 \%$, accuracy $85.6 \%$, positive and negative predictive values were $91.1 \%$ and $80.0 \%$ respectively for prediction of LM significant disease.

\section{Conclusion:}

From this study it may be concluded that ECG evaluation for ST segment deviation in lead aVR greater than that in lead V1 is supposed to be a positive predictor of left main coronary artery obstruction with highly sensitive and accurate. The present study showed the precordial leads V1 and V6 can predict the critical LMCA obstruction in patients with acute coronary syndrome. Patients with LM disease presents are mostly male smoker, usually present with STEMI with other coronary artery involvement and more frequently develop LVF.

\section{Study limitations}

Although the results of this study support the hypothesis, there are some facts to be considered which might affect the results. Relatively a small number of patients were included because acute left main coronary artery obstruction is not common. This study is only carried out in a selected group of patient i.e. patients with ACS but patients with chronic stable angina are not included.

\section{Recommendations:}

A multicentric study with large sample size should be undertaken to generalize the finding. The study findings should be used with caution until validated by larger multicentric study.

Conflict of Interest - None.

\section{References}

1. Chen Y, Jao YTFN, Fang CC, Yu CL, Chen C and Wang SP. Recognition of Left Main Occlusion in Acute Coronary Syndrome. Acta Cardiol Sin 2004; 20: 139-146.

2. Chikwe J, Kim M, Goldstone AB, Fallahi A and Athanasiou T. Current diagnosis and management of left main coronary disease. Eur J Cardio-Thorac 2010; 38: $420-430$.

3. Aygul N, Ozdemir K, Tokac M, Aygul MU, Duzenli MA, Abaci A, et al. Value of lead aVR in predicting acute occlusion of proximal left anterior descending coronary artery and in-hospital outcome in ST-elevation myocardial infarction: an electrocardiographic predictor of poor prognosis. J Electrocardiol 2008; 41(4): 335-341.

4. Gorgels APM, Engelen DJM and Wellens HJJ. Lead aVR, a mostly ignored but very valuable lead in clinical electrocardiography. J Am Coll Cardiol 2001; 38(5): 1355-1356.

5. Nikus KC and Eskola MJ. Electrocardiogram patterns in acute left main coronary artery occlusion. $J$ Electrocardiol 2008; 41(6): 626-629.

6. Yamaji H, Iwasaki K, Kusachi S, Murakami T, Hirami $\mathrm{R}$, Hamamoto $\mathrm{H}$, et al. Prediction of acute left main coronary artery obstruction by 12-lead electrocardiography. J Am Coll Cardiol 2001; 38(5): 1348-1354.

7. Ockene IS, Matthews CE, Rifai N, Ridker PM, Reed G, Stanek E. Variability and Classification Accuracy of Serial High-Sensitivity C-Reactive Protein Measurements in Healthy Adults. Clin Chem 2001; 47: 3444-3450.

8. Gonzalez-Porras JR, Martin-Herrero F and Garcia-Sanz R. Hyper-homocysteinemia is a risk factor of recurrent coronary event in young patients irrespective to the MTHFR C677T polymorphism. Thromb Res 2007; 119: 691-698.

9. D’Ascenzo F, Presutti DG, Picardi E, Moretti C, Omede“ $\mathrm{P}$, Sciuto F, et al. Prevalence and non-invasive predictors of left main or three-vessel coronary disease: evidence from a collaborative international meta-analysis including 22740 patients. Heart 2012; 98: 914-919.

10. Barrabés JA, Figueras J, Moure C, Cortadellas J and Soler-Soler J. Prognostic value of lead aVR in patients with a first non-ST-segment elevation acute myocardial infarction. Circulation 2003; 108: 814-819.

11. Yildirim N, Arat N, Doðan MS, Sökmen Y and Ozcan F. Comparison of traditional risk factors, natural history and angiographic findings between coronary heart disease patients with age $<40$ and $>$ or $=40$ years old. Anadolu Kardiyol Derg 2007; 7(2): 124-127. 
12. Avezum A, Makdisse M, Spencer F, Gore JM, Keith AA and Fox MB. Impact of age on management and outcome of acute coronary syndrome: Observations from the Global Registry of Acute Coronary Events (GRACE). Am Heart J 2005; 149(1): 67-73.

13. Rostoff P, Piwowarska W, Konduracka E, Libionka A, Bobrowska JM, Stopyra K, et al. Value of lead aVR in the detection of significant left main coronary artery stenosis in acute coronary syndrome. Kardiol Pol 2005; 62(2): 128-135.

14. Kosuge M, Kimura K, Ishikawa T, Ebina T, Shimizu T, Hibi K, et al. Predictors of left main or three-vessel disease in patients who have acute coronary syndromes with non-ST-segment elevation. Am J Cardiol 2005; 95(11): 1366-1369.

15. Aygul N, Salamov E, Dogan U and Tokac M. Acute occlusion of the left main trunk presenting as ST- elevation acute coronary syndrome. J Electrocardiol 2009; 43 (2010): 76-78.

16. Wong CK, Gao W, Stewart RA, French JK, Aylward PE and White HD. The prognostic meaning of the full spectrum of aVR ST-segment changes in acute myocardial infarction. Eur Heart J 2012; 33(3): 384392.

17. Uthamalingam S, Zheng, Leavitt HM, Pomerantsev E, Ahmado I, Gurm GS, et al. Exercise-induced st-segment elevation in ecg lead aVR is a useful indicator of significant left main or ostial lad coronary artery stenosis. J Am Coll Cardiol 2011; 4(2): 176-185.

18. Taggart DP, Kaul S, Boden WET, Ferguson B, Guyton RA, Mack MJ, et al. Revascularization for unprotected left main stem coronary artery stenosis. J Am Coll Cardiol 2008; 51(9): 885-891. 